\title{
SISMICIDAD EN COSTA RICA DURANTE EL 2014
}

\author{
SEISMICITY IN COSTA RICA DURING 2014
}

\author{
Juan L. Porras, Lepolt Linkimer, Rafael Barquero, María C. Araya, \\ Wilfredo Rojas, Oscar H. Lücke \& Magda Taylor
}
Red Sismológica Nacional (RSN: UCR-ICE), Apdo. 214-2060, San Pedro, Costa Rica *Autor para contacto: lepolt.linkimer@ucr.ac.cr

(Recibido: 4/05/2015; aceptado: 02/09/2015)

\begin{abstract}
During 2014, the National Seismological Network (RSN: UCR-ICE) located 6836 earthquakes and reported 219 felt events. There were six significant earthquakes with magnitude $\geq 6.0$. The majority $(83 \%)$ of felt earthquakes were shallow $(<30 \mathrm{~km})$ and $74 \%$ had magnitudes $(\mathrm{Mw})$ between 3.0 and 4.9. Local and regional faulting caused $64 \%$ of the felt earthquakes and the subduction of the Cocos Plate beneath the Caribbean Plate and the Panama microplate caused 29\%. The highest intensity observed during 2014 was VI (Modified Mercalli) on the Burica peninsula during the December 8 earthquake (6.1 Mw) with the epicenter at western Panama.

Keywords: Seismicity, seismic sources, Seismology, subduction, seismic intensity.

RESUMEN: Durante el 2014 la Red Sismológica Nacional (RSN: UCR-ICE) localizó 6836 sismos de los cuales 219 fueron sentidos por la población. Se percibieron en Costa Rica seis sismos con magnitud Mw $\geq 6,0$. La mayoría $(83 \%)$ de los sismos percibidos fueron superficiales $(<30 \mathrm{~km})$ y el $74 \%$ tuvo una magnitud $(\mathrm{Mw})$ de entre 3,0 y 4,9 . El fallamiento local y regional provocó el $64 \%$ de los sismos, seguido por el proceso de subducción de la placa del Coco bajo la placa Caribe y la microplaca de Panamá que originó el 29\%. La intensidad máxima observada en el 2014 fue de VI (Mercalli Modificada) en la península de Burica debido al sismo del 8 de diciembre (Mw 6,1) cuyo epicentro se localizó en el oeste de Panamá.

Palabras clave: Sismicidad, fuentes sísmicas, Sismología, subducción, intensidad sísmica.
\end{abstract}




\section{INTRODUCCIÓN}

En el 2014 la Red Sismológica Nacional (RSN: UCR-ICE) localizó 6836 sismos de los cuales 219 fueron percibidos por la población (Fig. 1, 2 y 3.A). La cantidad de sismos localizados en el año incluye sismos ocurridos en el territorio de Costa Rica, América Central y distantes (telesismos). El 2014 es el cuarto año con mayor cantidad de sismos sentidos en el registro de la RSN. Desde 1976, los años con la mayor cantidad de sismos sentidos han sido 1991, 2012 y 2013 con 353, 264 y 261, respectivamente (Linkimer \& Alvarado, 2014). El promedio anual de sismos sentidos para el periodo 1976-2014 es de 90 (Linkimer \& Alvarado, 2014).

La Red Sismológica Nacional (RSN) fue creada mediante un convenio entre la Sección de Sismología, Vulcanología y Exploración Geofísica de la Escuela Centroamericana de Geología de la Universidad de Costa Rica (UCR) y el Área de Amenazas y Auscultación Sísmica y Volcánica del Instituto Costarricense de Electricidad (ICE). La sede y centro de registro de la RSN se localiza en la Escuela Centroamericana de Geología de la UCR y funciona desde 1973. Los sismos localizados durante el 2014, fueron registrados por una red de 47 estaciones sismológicas ubicadas en diferentes partes de Costa Rica y unas 19 estaciones internacionales ubicadas en Panamá y Nicaragua. La detección y localización de los sismos localizados durante el 2014 se realizó a través de los sistemas Seiscomp, Earthworm y SeisAn. La lectura de arribos de ondas, localización y cálculo de magnitudes, se llevó a cabo manualmente mediante el programa HYP (Lienert \& Havskov, 1995) y la última versión del software sismológico SeisAn (Ottemöller et al., 2011). Para el proceso de localización se empleó un modelo de velocidades de la onda Primaria $(\mathrm{P})$ de siete capas, una razón de velocidades $(\mathrm{Vp} / \mathrm{Vs})$ de 1,74 y se calculó la magnitud momento (Mw) para cada sismo.

\section{CARACTERÍSTICAS DE LOS SISMOS SENTIDOS}

A continuación se describe la sismicidad que fue percibida por la población de Costa Rica durante el 2014 tomando en cuenta la distribución temporal, profundidad, magnitud $(\mathrm{Mw})$ y origen.

\section{Distribución temporal}

El promedio mensual de sismos sentidos para el año 2014 fue de 18. Los meses con mayor cantidad de sismos sentidos fueron marzo y agosto con 28 y 22, respectivamente (Fig. 3A). Los meses con la menor cantidad de sismos sentidos fueron febrero y septiembre, en los que se reportaron solamente 11 y 10 sismos, respectivamente. El resto de meses del año presentaron una cantidad de sismos que varió entre 13 y 21 (Fig. 3A).

\section{Distribución por magnitud (Mw)}

El sismo sentido de mayor magnitud del 2014 ocurrió el 13 de octubre, de Mw 7,3 y se localizó frente a la costa del Golfo de Fonseca, 67 $\mathrm{km}$ al suroeste de Jiquilillo, Nicaragua. Cinco sismos sentidos más tuvieron magnitudes mayores a 6,0 durante el 2014. Todos estos sismos estuvieron ubicados fuera de los límites de Costa Rica, por ejemplo los ocurridos al sur de la provincia de Chiriquí en Panamá el 13 de mayo, 6 de diciembre y 8 de diciembre, con Mw de 6,5, 6,0 y 6,1 , respectivamente y en Nicaragua los días 2 de marzo y 10 de abril con $\mathrm{Mw}$ de 6,2 y 6,1, respectivamente.

Los sismos sentidos de mayor magnitud ubicados dentro del territorio costarricense ocurrieron el 13 de octubre y 16 de noviembre, ambos con $\mathrm{Mw}$ 5,3 . Estos eventos se ubicaron a $37 \mathrm{~km}$ al suroeste de Bahía Ballena de Osa y a $43 \mathrm{~km}$ al oeste de Cabo Velas en Guanacaste, respectivamente (Fig. 2). 


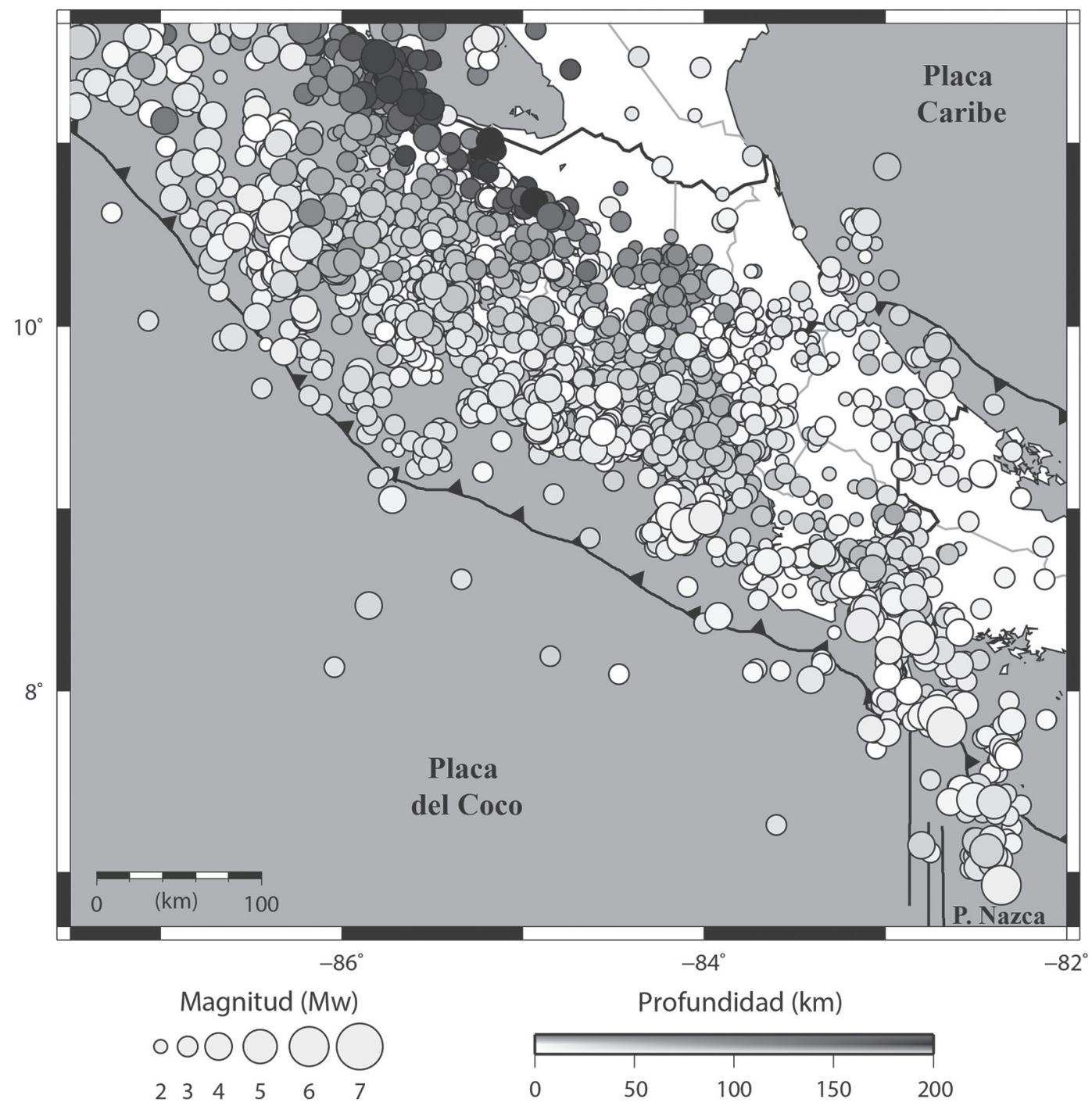

Fig. 1: Ubicación epicentral de los sismos localizados por la RSN durante el año 2014.

Otros dos sismos sentidos de magnitud sobresaliente (Mw 5,2) ocurrieron el 17 de enero y el 6 de julio y se ubicaron $29 \mathrm{~km}$ al oeste de Jacó y $1 \mathrm{~km}$ al noroeste de Quebrada Honda en Nicoya, respectivamente. El 12\% de los sismos sentidos del 2014 tuvieron magnitudes que oscilaron entre 5,0 y 7,3. La mayoría $(74 \%)$ de los sismos percibidos tuvieron una $\mathrm{Mw}$ entre 3,0 y 4,9 (Fig. 3B). El sismo de menor magnitud del 2014 que fue reportado como sentido ocurrió el 1 de noviembre, tuvo una Mw de 2,4 y se localizó 


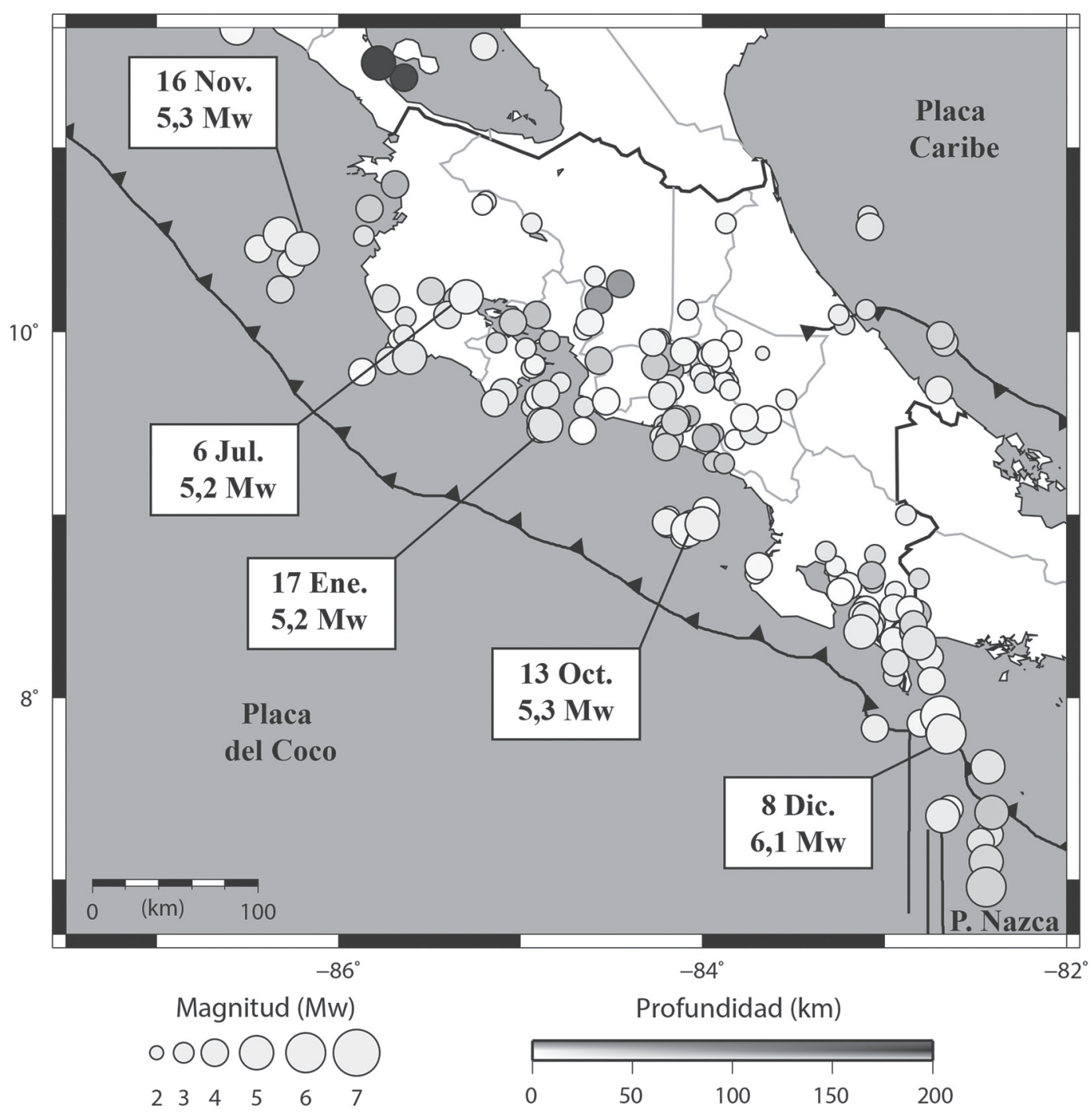

Fig. 2: Ubicación epicentral de los sismos sentidos durante el 2014 en Costa Rica.

$2 \mathrm{~km}$ al noroeste de San Miguel de Desamparados, al sur de San José. En total 31 sismos de baja Mw $(<2,9)$ fueron percibidos por la población (Fig. 3B) y estos en su mayoría tuvieron epicentros muy cerca de zonas urbanas como Alajuelita, Desamparados, Cartago y La Guácima de Alajuela.

\section{Distribución por profundidad}

El 83\% de los sismos sentidos tuvieron profundidades menores a $30 \mathrm{~km}$ (Fig. 3C). La mayoría de los sismos tuvieron profundidades menores a $9,9 \mathrm{~km}(101$ sismos) y entre 10 y 19,9 km (54 

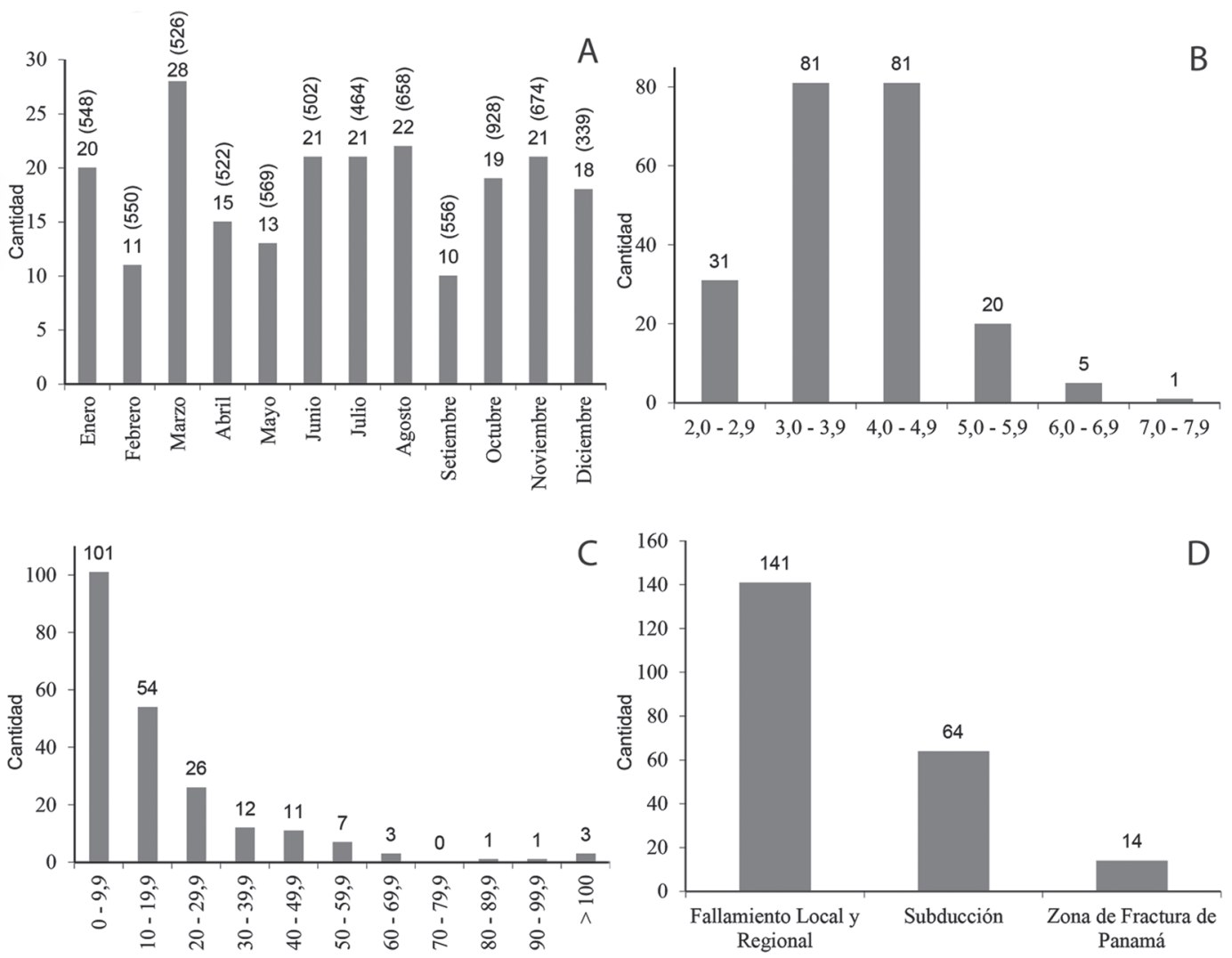

Fig. 3: Distribución de los sismos sentidos del 2014 por A) mes B) magnitud C) profundidad y D) origen. La cantidad de sismos sentidos se muestra sobre la barra de cada histograma y la cantidad de sismos localizados se muestra entre paréntesis.

sismos). Treinta y ocho sismos sentidos tuvieron su hipocentro a una profundidad mayor de $30 \mathrm{~km}$. Estos sismos están asociados con la deformación interna de la placa del Coco que se subduce debajo de la placa Caribe y la microplaca de Panamá. Los sismos de profundidad intermedia, esto es, de entre 30 y $300 \mathrm{~km}$, no suelen ser percibidos por la población a menos que sean de una magnitud intermedia $o$ alta.

El sismo sentido más profundo del 2014 ocurrió el 6 de octubre, tuvo una magnitud de 5,0 y se localizó $33 \mathrm{~km}$ al noroeste de Peñas
Blancas en Guanacaste a una profundidad de $189 \mathrm{~km}$. Otros tres sismos sentidos tuvieron una profundidad de entre 92 y $184 \mathrm{~km}$, ocurrieron en Nicaragua (11 de abril, Mw 5,9 y profundidad $125 \mathrm{~km}$ y 4 de agosto, Mw 4,6 y profundidad $184 \mathrm{~km}$ ) y en Tapesco de Zarcero (25 de marzo, $\mathrm{Mw} 4,2$ y profundidad $92 \mathrm{~km}$ ). El sismo sentido de mayor tamaño de profundidad intermedia ocurrió el 2 de marzo, tuvo una Mw de 6,2 y se localizó en las cercanías de Chinandega en Nicaragua a una profundidad de $67 \mathrm{~km}$. 


\section{Distribución por origen}

La mayoría de los sismos sentidos del 2014 (64\%) fueron originados en fallas locales y regionales (Fig. 3D). Esto incluye los sismos superficiales $(<30 \mathrm{~km})$ ocurridos dentro de la placa Caribe y la microplaca de Panamá y en las fallas del Cinturón Deformado del Norte de Panamá (Adamek et al., 1988) y Cinturón Deformado del Centro de Costa Rica (Marshall et al., 2000). Otro porcentaje importante de los sismos sentidos (29\%) ocurrió debido al proceso de subducción de la placa del Coco bajo la placa Caribe y la microplaca de Panamá (Fig. 3D). Este proceso incluye los sismos ocurridos en la zona interplacas y los de profundidad intermedia (>50 km) que se asocian con la deformación interna de la placa del Coco que se subduce bajo Costa Rica. Adicionalmente, catorce sismos fueron originados en la Zona de Fractura de Panamá, que corresponde con el límite entre las placas del Coco y Nazca (Fig. 3D).

\section{Intensidades}

El sismo que generó las intensidades más altas del 2014 dentro de Costa Rica ocurrió el 8 de diciembre, $77 \mathrm{~km}$ al Sur de Laurel de Corredores con una Mw 6,1 (Fig. 2). Este sismo fue percibido con intensidades (MM) de hasta VI en poblados de la Península de Burica, así como en Puerto Armuelles en Panamá. Además fue reportado con intensidad V en David, El Progreso y Concepción en Panamá y del lado costarricense en Laurel, Puerto Jiménez, Ciudad Neily y Golfito. También fue sentido con intensidad IV en San Isidro de Pérez Zeledón, San Vito de Coto Brus y Ciudad Cortés.

El segundo sismo más significativo por las intensidades generadas ocurrió el 6 de julio cerca de Quebrada Honda de Nicoya (Mw 5,2). Para este evento se observaron intensidades de hasta V en Nicoya, Atenas, San Ramón, Zarcero, Naranjo, Ciudad Quesada, Miramar y Barranca de Puntarenas.

Los sismos sentidos de mayor magnitud del año ocurrieron fuera de los límites de Costa Rica y por su alta magnitud fueron percibidos en nuestro país. Por ejemplo, el terremoto del Golfo de Fonseca del 13 de octubre (Mw 7,3) fue percibido con intensidad (MM) III en gran parte de Costa Rica y el sismo en la Bahía de Charco Azul en Panamá del 13 de mayo (Mw 6,5) se reportó sentido con intensidad IV en Laurel, Canoas y Golfito y III en Turrialba, Limón y el Valle Central.

Los sismos sentidos de mayor magnitud ubicados dentro del territorio costarricense ( $\mathrm{Mw} 5,3$ ) ocurrieron el 13 de octubre y 16 de noviembre al suroeste de Bahía Ballena de Osa y al oeste de Cabo Velas en Guanacaste, respectivamente (Fig. 2). Estos sismos fueron percibidos con intensidad de IV en Dominical, Quepos, Golfito y Bahía Drake (13 de octubre) y en los poblados de Playa Conchal, Brasilito, Huacas y Cartagena de Santa Cruz (16 de noviembre).

Varios sismos de magnitudes bajas pero con epicentros cercanos al Área Metropolitana de San José fueron ampliamente sentidos. Estos sismos ocurrieron el 24 de Julio (4,6 Mw) cerca La Guácima de Alajuela, el 25 de Julio (4,5 Mw) al norte de Cartago, el 7 de agosto (4,6 Mw) cerca de San Carlos de Tarrazú y el 1 de noviembre (4,3 Mw) cerca de Alajuelita. Estos sismos fueron observados con intensidades de hasta $\mathrm{V}$ en las zonas epicentrales.

En las zonas de Esparza y Pavón de Golfito se presentaron también sismos sentidos relevantes. En el caso de Esparza, estos ocurrieron principalmente durante los meses de abril y mayo. El sismo de mayor magnitud $(4,4 \mathrm{Mw})$ ocurrió el 25 de abril y fue sentido con intensidad IV en Puntarenas y Esparza y III en el Valle Central. En el caso de Pavón de Golfito los sismos ocurrieron en octubre, con el sismo principal el día 10 de $(5,1$ $\mathrm{Mw}$ ). Este sismo fue sentido con intensidad $\mathrm{V}$ en Golfito, Puerto Jiménez, Río Claro y Paso Canoas. 


\section{AGRADECIMIENTOS}

A todo el personal de la RSN en la Escuela Centroamericana de Geología de la Universidad de Costa Rica (UCR) y en el Área de Amenaza y Auscultación Sísmica y Volcánica del Instituto Costarricense de Electricidad (ICE).

\section{REFERENCIAS BIBLIOGRÁFICAS}

ADAMEK, S.,FROHLICH, C.\&PENNINGTON, W., 1988: Seismicity of the CaribbeanNazca Boundary Constraints on Microplate Tectonics of the Panama Region.- J. Geophys. Res. 93: 2053-2075.

LIENERT, B. R. \& HAVSKOV, J., 1995: A Computer Program for Locating Earthquakes Both Locally and Globally.- Seis. Res. Lett. 66: 26-36, DOI: 10.1785/gssrl.66.5.26.
LINKIMER, L. \& ALVARADO, G. E., 2014: Distribución espacio-temporal de la sismicidad sentida en Costa Rica (1976-2013) en el marco histórico del 30 aniversario (1982-2012) de la Red Sismológica Nacional (RSN: UCR-ICE).- Rev. Geol. Amér. Central, Vol. Esp. 30 aniversario: 45-71.

MARSHALL, J. S., FISHER, D. M. \& GARDNER, T. W., 2000: Central Costa Rica deformed belt: Kinematics of diffuse faulting across the western Panama block.Tectonics, 19, 468-492.

OTTEMÖLLER, L., VOSS, P. \& HAVSKOV, J., 2011: SEISAN: The Earthquake Analysis Software for Windows, Solaris, LINUX, and MACOSX, version 9.0.1.- 361 págs. Univ. of Bergen, Bergen. 
